\title{
Age differences in default mode activity on easy and difficult spatial judgment tasks
}

\author{
Denise C. Park ${ }^{1}$, Thad A. Polk ${ }^{2}$, Andrew C. Hebrank ${ }^{1}$ and Lucas J. Jenkins ${ }^{3}$ \\ 1 Center for Vital Longevity, University of Texas at Dallas, Dallas, TX, USA \\ 2 Department of Psychology, University of Michigan, Ann Arbor, MI, USA \\ ${ }^{3}$ Department of Psychology, University of California Davis, Davis, CA, USA
}

\section{Edited by:}

William J. Jagust, University of

California Berkeley, USA

\section{Reviewed by:}

Reisa Sperling, Harvard University, USA Michael Greicius, Stanford University

School of Medicine, USA

\section{*Correspondence:}

Denise C. Park, Center for Vital

Longevity, 2200 West Mockingbird

Lane, Dallas, TX 75235, USA.

e-mail:denise@utdallas.edu
The default network is a system of brain areas that are engaged when the mind is not involved in goal-directed activity. Most previous studies of age-related changes in default mode processing have used verbal tasks. We studied non-verbal spatial tasks that vary in difficulty. We presented old and young participants with two spatial judgment tasks: an easy categorical judgment and a more demanding coordinate judgment. We report that (a) Older adults show markedly less default network modulation than young on the demanding spatial task, but there is age equivalence on the easy task; (b)This Age $\times$ Task interaction is restricted to the default network: Brain areas that are deactivated by the tasks, but that are outside the default network, show no interaction; (c) Young adults exhibit significantly stronger functional connectivity among posterior regions of the default network compared with older adults, whereas older adults exhibit stronger connectivity between medial prefrontal cortex and other sites; and (d) The relationship of default activity to reaction time performance on the spatial tasks is mediated by age: in old adults, those who deactivate the default network most also perform best, whereas the opposite is true in younger adults. These results extend the findings of age-related changes in default mode processing and connectivity to visuo-spatial tasks and demonstrate that the results are specific to the default network.

Keywords: default mode, deactivation, aging brain, spatial judgment, parietal cortex, connectivity, fMRI

\section{INTRODUCTION}

In the past decade, we have learned a great deal about the function of the aging mind by examining patterns of neural activity using neuroimaging. There are a number of major findings that characterize neural activation in older adults. For one, older adults, in contrast to younger adults, show broader swaths of activation in frontal cortex when performing attentional (Madden et al., 1999), working memory (Reuter-Lorenz et al., 2001), and longterm memory tasks (Cabeza et al., 2002). Frequently the observed pattern is one of frontal cortex bilaterality for old and unilaterality in young (Cabeza, 2002). There is good evidence (reviewed by Park and Reuter-Lorenz, 2009) that this additional frontal activation is compensatory, as it has been shown to be associated with remembered but not forgotten items in old (Gutchess et al., 2005), is more typical of high performers than low performers (Cabeza et al., 2002; Rosen et al., 2002), is associated with decreased hippocampal activation in old on a memory task (Gutchess et al., 2005), and its disruption via transcranial magnetic stimulation leads to poorer performance in older subjects (Rossi et al., 2004). A second major finding is that ventral visual cortex exhibits greater dedifferentiation in older adults compared to young. That is, older adults show less specificity of neural activity (Park et al., 2004; Chee et al., 2006), and tend to show a pattern of increased frontal/decreased ventral visual activation compared to young (Davis et al., 2008). Finally, there is a growing literature suggesting that older adults are both less efficient at suppressing default network activity when faced with cognitive challenge (Grady et al., 2006; Persson et al., 2007; Duverne et al., 2009) and show poorer interconnectivity among default sites (Andrews-Hanna et al., 2007; Miller et al., 2008).

The present paper focuses on task-related deactivations in areas collectively referred to as the default mode network. The default network is an organized network of brain sites that are engaged when the brain is in a resting state (Raichle et al., 2001), and is isolated in fMRI studies by examining interconnectivity among sites with subjects at rest or by contrasting baseline neural activity (e.g., staring at a fixation point) in a cognitive study with neural activations that occur under cognitive challenge (Shulman et al., 1997; Raichle and Snyder, 2007). The default network encompasses a range of neural sites including the medial frontal cortex, medial and lateral parietal cortex, and anterior and posterior cingulate, as well as medial temporal areas. Default activity does not represent a state of "no cognitive activity," but rather is characteristic of thought that is not goal-directed and may involve day dreaming, self-referential processing, or the simulation of future events (Mason et al., 2007; Schacter et al., 2007; Addis et al., 2008)'. It is clear that when young adults are faced with a cognitive challenge, the activity in the default sites is suppressed, and areas that

${ }^{1}$ We note that there is some debate about whether the default network represents conscious thought or merely represents intrinsic neural connectivity. Studies involving sleep, sedation, and patients in a vegetative state indicate the persistence of default activity in the absence of consciousness (Vincent et al., 2007; Greicius et al., 2008; Boly et al., 2009) so it remains (and may continue to remain) an open question whether default activity provokes a subjective experience. 
are associated with task performance are activated. Moreover, the magnitude of the suppression is increased as tasks become more difficult (McKiernan et al., 2003).

There are data suggesting that as people age, they become less efficient at suppressing the default network, particularly when faced with cognitive challenge (Grady et al., 2006; Andrews-Hanna et al., 2007; Persson et al., 2007; Duverne et al., 2009). There is also evidence that the default network is more poorly organized in older adults, showing weaker functional connectivity among default sites (Andrews-Hanna et al., 2007) as well as both poorer connectivity and poorer modulation of default activity in patients with Alzheimer's disease (Lustig et al., 2003; Greicius et al., 2004; Buckner et al., 2005). Poor modulation and lowered connectivity of default sites are linked to poor cognitive function in healthy older adults (Persson et al., 2007; Damoiseaux et al., 2008). To summarize, inefficient default mode activity and poor default network organization is characteristic of older adults and, in the extreme, is typical of patients with Alzheimer's disease.

One study has suggested that older adults are also less able to modulate default activity in response to changes in task difficulty. Persson et al. (2007) presented young and old adults with a verbfinding task that was constrained and easy (e.g., produce a verb to act on the noun "scissors") or less constrained with more alternatives (e.g., produce a verb to "ball"). They reported that as the task had fewer constraints and required more frontal processing, default suppression increased in young but not old, and they concluded that older adults are less able to modulate default activity in response to cognitive challenge.

Thus far, studies of default activity and aging have focused primarily on verbal encoding and retrieval tasks, and there is little work on the ability to modulate default activity as task demands change. In the present study, we examined the ability of healthy young and older adults to modulate default activity, as well as connectivity among sites, in non-verbal spatial tasks that activated a frontal-parietal network. Relatively little is known about age differences in parietal activity, although there is limited evidence that increased recruitment of parietal lobes occurs in old adults compared to young adults on cognitive tasks (Davis et al., 2008). In the present study, we utilized a categorical/coordinate judgment task with young and old adults that placed two levels of demand on parietal lobe function (Baciu et al., 1999). In the categorical task, subjects judge whether a dot is above or below a horizontal line. In the coordinate task, they make a referential judgment about whether the dot is further from the line than a standard distance held in memory. There is clear evidence that the coordinate task is more difficult than the categorical task in terms of neural activity as well as reaction times and error rates (see Jager and Postma, 2003 for a review). Some studies find that the coordinate task is more likely to show right parietal activations whereas the categorical task is more biased toward left parietal activity, but other studies report mixed evidence for such differences in laterality (Jager and Postma, 2003; Martin et al., 2008).

We conducted the present study to examine age differences in fronto-parietal activations on a visuo-spatial task, and to assess age differences in both connectivity and modulation of the default network as task difficulty increased. We hypothesized that young adults would show increasing suppression of default network sites under challenge, whereas old would not show suppression that was graded by task difficulty. We also expected greater interconnectivity among default sites in young compared to old. We further hypothesized that older subjects who performed well on the judgment task would show the most effective modulation of default activity. Finally, we predicted that old would show increased bilateral recruitment of frontal and parietal areas relative to young as the task became more difficult.

\section{MATERIALS AND METHODS PARTICIPANTS}

All study procedures were reviewed and approved by the University of Illinois Institutional Review Board, and all participants provided detailed written consent prior to their involvement in this study. We recruited 23 younger adults from the University of Illinois. Of this sample, 19 (ages 19-30; 10 female) yielded usable datasets and 4 were discarded because of excessive motion during functional imaging. We also tested 24 healthy community-dwelling older adults from the Champaign-Urbana area (ages 61-69, 10 female). We discarded five older participants because of excess motion (one participant), distortion due to improper headcoil placement (two participants), or vision problems and/or failure to follow instructions (two participants). All participants were screened for eligibility (including being right-handed, a native English speaker, and psychologically and physically healthy; not taking medications with psychotropic or vascular effects; and free of any other MRI safety contraindicators). Old and young subjects had equivalent levels of education and verbal ability as measured on the Shipley Institute of Living Scale (Zachary, 1986), as shown in Table 1. Subjects had a minimum score of 26 on the Mini Mental Status Examination (Folstein et al., 1975), with mean scores for young and old of 28.8 and 27.8 respectively, a difference that was significant $[t(36)=2.069, p<0.05]$. Older adults also evidenced lower scores

Table 1 | Participant characteristics.

\begin{tabular}{lcc}
\hline \multicolumn{1}{c}{ Young } & Old \\
\hline PARTICIPANT DEMOGRAPHIC AND PSYCHOMETRIC & \\
CHARACTERISTICS & \\
$N$ & 19 & 19 \\
Female (M) & 10 & 10 \\
Age (years) & $22.2(2.4)$ & $64.8(2.8)^{*}$ \\
Education (years) & $15.0(1.3)$ & $15.9(2.9)$ \\
MMSE & $28.8(1.0)$ & $27.8(1.7)^{*}$ \\
Digit symbol & $74.2(10.7)$ & $52.5(8.4)^{*}$ \\
Shipley vocabulary & $33.6(3.1)$ & $34.4(4.1)$ \\
fMRI TASK BEHAVIORAL CHARACTERISTICS & \\
Task accuracy & & $0.87(0.2)$ \\
Categorical & $0.93(0.0)$ & $0.72(0.1)^{*}$ \\
Coordinate & $0.83(0.1)$ & $653.0(99.4)^{*}$ \\
Task RT (ms) & & $847.6(92.8)^{*}$ \\
Categorical & & \\
Coordinate & $540.7(74.3)$ &
\end{tabular}

Mean values or counts with standard deviations. Mean values differing significantly by age $(p<0.05)$ are indicated with asterisks. 
on the WAIS Digit-Symbol Test (Wechsler, 1981), which measured speed of processing $[t(36)=6.97, p<0.001]$. This pattern of stable crystallized ability with age (vocabulary) and lower fluid abilities (speed) is typical of most cognitive aging samples in the literature that focus on normal aging (Park et al., 2002).

\section{FUNCTIONAL IMAGING}

Prior to entering the scanner, all participants underwent a short training session and practiced modified versions of the spatial judgment tasks used by Baciu et al. (1999). The tasks included a categorical task, a coordinate task, and a baseline task (Figure 1). In the categorical condition, participants viewed a series of stimuli in which a dot appeared near a horizontal bar. The participant's task was to judge whether the position of the dot was above the horizontal bar by pressing either the "yes" or "no" key with their finger. In the coordinate task, participants first viewed a vertical reference line at the beginning of a block of stimuli. When they viewed each subsequent stimuli, which consisted of a horizontal bar with a dot above or below it, they were to judge whether the dot above or below the bar was farther away from the horizontal bar than the length of the reference line viewed at the beginning of the block. In both of these conditions, dots could appear in any one of 12 predefined distances, with half above and half below the horizontal bar. Finally, a control condition consisted of a horizontal bar between two dots (designed to prevent participants from inadvertently performing categorical judgments during this condition). Participants were asked to respond with alternating button presses each time a stimulus of this type appeared.

The tasks were presented in the magnet in blocks of 15 items. Each block began with a 6-s cue that indicated the task to perform. The word "ABOVE?" cued participants to perform the categorical judgment task on the subsequent stimuli. "FARTHER?" cued performance of the coordinate judgment, and "ALTERNATE" cued performance of the response-matched control condition. Each stimulus was presented for $1750 \mathrm{~ms}$ with a $250-\mathrm{ms}$ ISI. There were 12 blocks, presented in pseudorandom order in a run, with a run lasting 442 s (12 36-s stimulus blocks plus a 10-s delay at the beginning of the run). There were two runs.

Stimuli were presented via E-prime (Psychology Software Tools, Pittsburgh, PA, USA) and displayed by a back-projection system. Participants made responses using buttons under the index and middle fingers of the right hand (Lumina response pad; Cedrus, San Pedro, CA, USA). A "yes" response was made with the index finger and a "no" response with the middle finger. Information about decision response time and accuracy was recorded during each stimulus presentation.

\section{MR ACOUISITION}

Brain imagery was acquired using a 3T Siemens Allegra head-only system (Siemens; Erlanger, Germany). Functional images were collected using a conventional echo-planar MR protocol, with complete volumes acquired every $2 \mathrm{~s}\left(\mathrm{TE}=25 \mathrm{~ms}, \mathrm{FA}=80^{\circ}, \mathrm{FOV}=220 \mathrm{~mm}\right)$. Slices were $64 \times 64$ matrices acquired parallel to the AC-PC line. Each volume consisted of 36 slices spanning $158 \mathrm{~mm}$ and encompassing all of the cerebrum and most of the cerebellum. In addition to functional imagery, a high-resolution ( $1 \mathrm{~mm}$ isotropic voxels) T1-weighted MPRAGE was also acquired, primarily to facilitate warping individual volumes to a shared space.

\section{DATA PROCESSING}

Using routines from SPM5 (Wellcome Department of Cognitive Neurology, London, UK), images were corrected for temporal offset of slice acquisition. From motion estimates, an affine transformation was then applied to each volume. Each individual's images were warped to a standard space (MNI), resampled to $3 \mathrm{~mm}$ isotropic voxels, and spatially smoothed with an 8-mm FWHM Gaussian kernel.

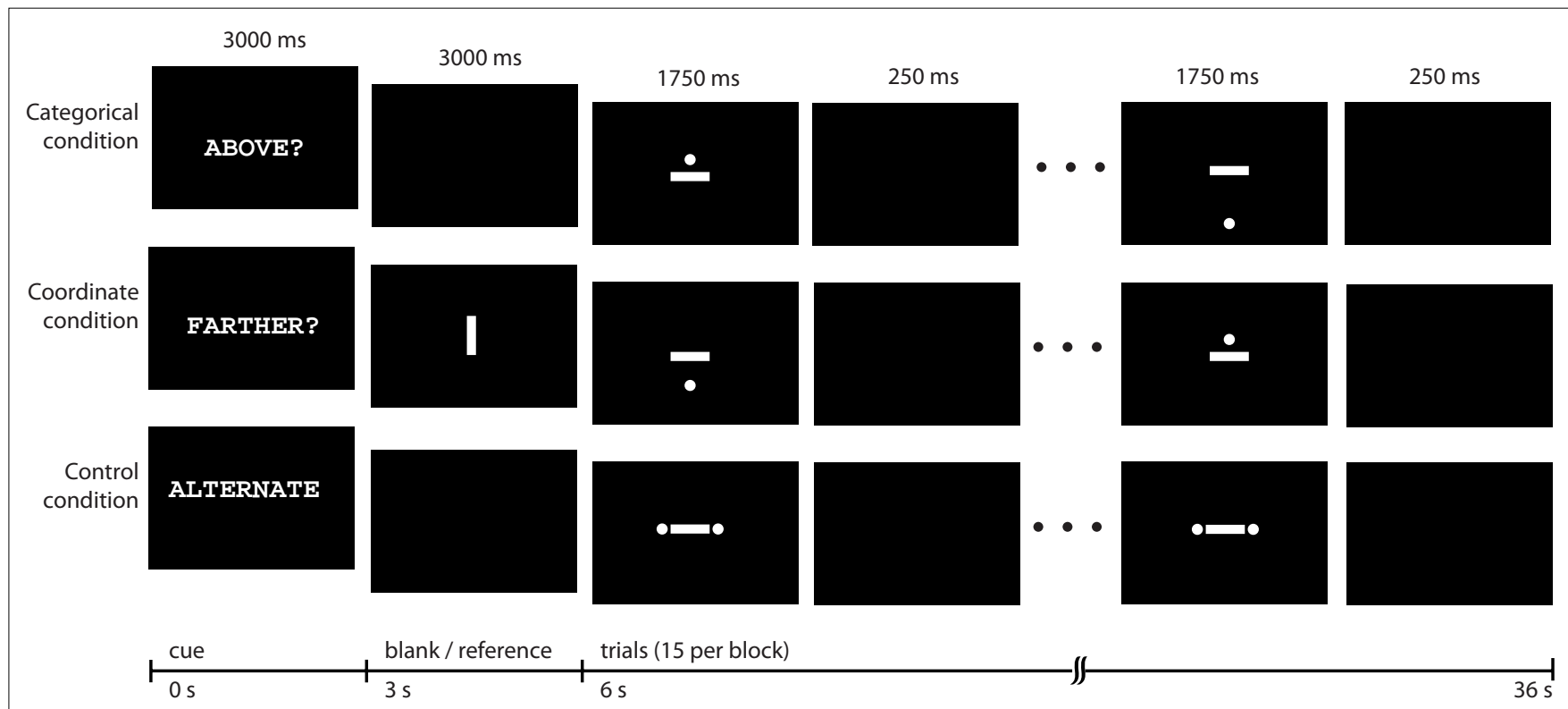

FIGURE 1 |Task conditions. Categorical, coordinate, and control conditions each occurred in 36-s blocks that were presented in pseudorandom order. 
To generate individual statistical maps, stimulus blocks were first convolved with a canonical HRF. The resulting regressors (one for each of the three task conditions) were modeled along with motion estimates and a regressor corresponding to each run/scan (to model differences in mean signal across scans). Arithmetic combinations of parameter estimates were used to compute contrast maps for each participant. Additionally, these contrast maps were entered into second-level group analyses that regressed on age group membership.

\section{CONNECTIVITY}

We also examined connectivity among different sites in the default network. To avoid sampling bias, regions within the default network were taken from prior work (Andrews-Hanna et al., 2007) and were defined as $4 \mathrm{~mm}$ spheres centered on points in the left and right lateral parietal cortex (MNI -46 -70 25 and 53-65 26, respectively), posterior cingulate and retrosplenial cortex (MNI 1 -53 26), the hippocampus (MNI -23-25-16; $23-25-16)$ and parahippocampal cortex (MNI $-25-40-14 ; 25-40-14$ ), and medial prefrontal and anterior cingulate cortex (MNI 140 20). Functional data were processed following established procedures (Fox et al., 2005) to remove effects of no interest from the fitted models. Specifically, each voxel's signal time series was temporally filtered to remove frequencies higher that $0.08 \mathrm{~Hz}$ and lower than $0.009 \mathrm{~Hz}$. Additional effects removed included: a six-parameter estimate of head motion, mean whole-brain signal, signal from a region centered in deep white matter, signal from a region within the lateral ventricles, and signal associated with convolved categorical and coordinate task regressors (leaving only signal associated with the control task). For each of the regions of interest (ROI), the resulting residual time series (averaged across voxels within a region) was correlated with the residual time series in every other region, resulting in an interregion connectivity matrix. Because removing global signal results in a distribution shift of correlation coefficients (Vincent et al., 2006), we restrict interpretation of this matrix to positive correlations differing significantly from $r=0$.

\section{RESULTS \\ BEHAVIORAL RESULTS}

Table 1 presents the behavioral results. An analysis of variance of RT with Age and Task Type (Coordinate or Category) as factors yielded main effects of both Age $[F(1,72)=31.31, p<0.001]$ and Task Type $[F(1,72)=96.65, p<0.001]$ with no interaction. The young adults were significantly faster than the old adults. A similar ANOVA conducted on accuracy also yielded main effects of Age $[F(1,72)=9.92, p<0.05]$ and Task Type $[F(1,72)=22.87$, $p<0.001]$. We also note that two-tailed $t$-tests indicated that the categorical task was significantly easier than the coordinate task for both young and old subjects. Accuracy was higher in the categorical task than in the coordinate task [young: $t(18)=8.24, p<0.001$; old: $t(18)=4.85, p<0.001$ ] and reaction times were faster [young: $t(18)=15.42, p<0.001$; old: $t(18)=11.10, p<0.001]$.

\section{IMAGING RESULTS}

\section{Whole-brain analyses}

Figure 2 and Table 2 show the results of a group analysis of the imaging data from all the subjects, young and old combined $(p<0.05$ after correcting for family-wise error across the whole brain). For

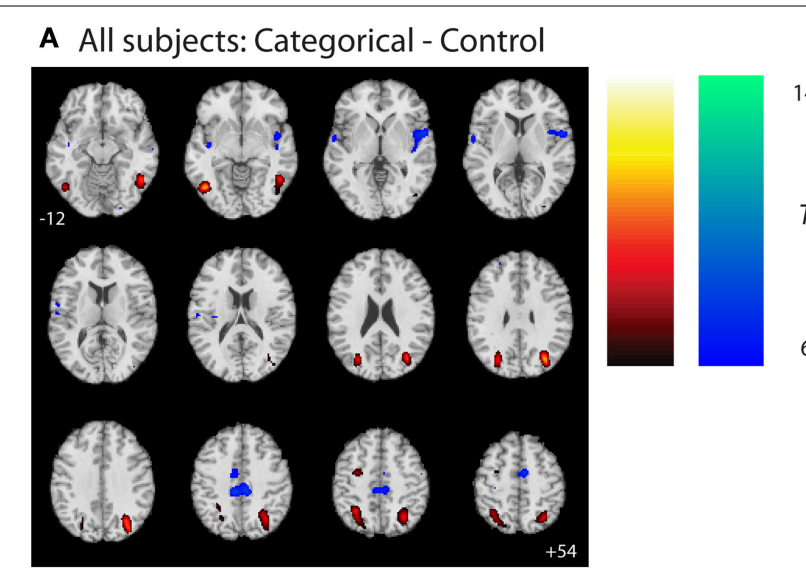

14

$T$

B All subjects: Coordinate - Control

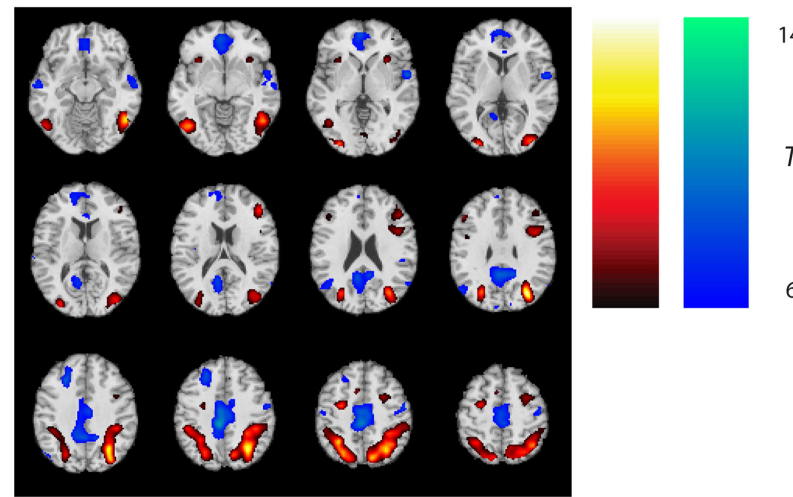

C All subjects: Coordinate - Categorical

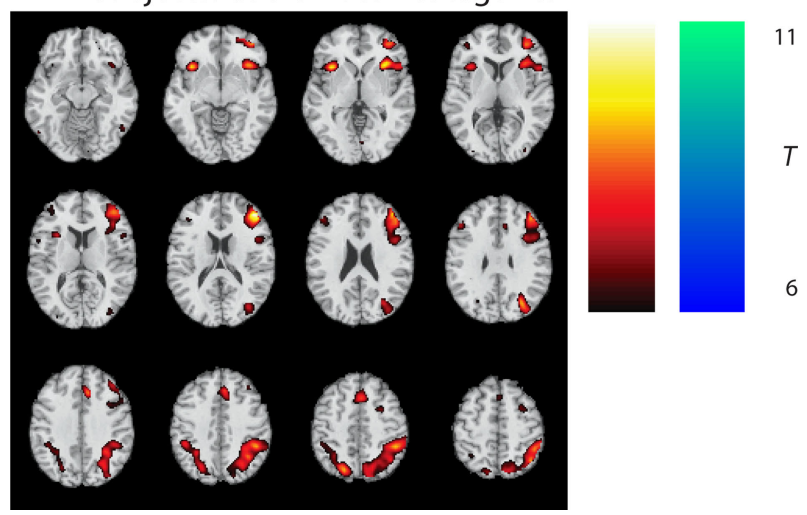

FIGURE 2 | Group statistical maps, all subjects. (A) Categorical-control task T-map for young and old grouped together. (B) Coordinate-control task T-map for young and old together. (C) Coordinate-categorical tasks T-map for young and old combined. Note that the reverse contrast reveals no significant activation. All maps thresholded with FWE correction at $p<0.05$, and reverse contrasts shown in cold colors.

the easier categorical task (vs. the control task), the primary taskrelated activation sites were in posterior parietal cortex bilaterally and in ventral visual areas (left and right inferior temporal gyri, left and right middle occipital gyri) (Figure $2 \mathbf{A}$ and Table 2).

The more difficult coordinate task (vs. the control task) activated a superset of the areas activated by the categorical task, yielding considerably more task-related activation in the posterior parietal cortex (Figure 2B and Table 2). In addition, the coordinate task 
Table 2 |Top clusters from group statistical maps, all subjects.

\begin{tabular}{|c|c|c|c|}
\hline Region & $\begin{array}{l}\text { Cluster } \\
\text { extent }\end{array}$ & $\begin{array}{l}\text { MNI mm } \\
\text { (peak) }\end{array}$ & $\begin{array}{l}t \text { value } \\
\text { (peak) }\end{array}$ \\
\hline \multicolumn{4}{|c|}{ CATEGORICAL-CONTROLTASK FOR YOUNG AND OLDTOGETHER } \\
\hline \multicolumn{3}{|c|}{ R sup parietal, } & \\
\hline R inf temporal & 646 & $33-7230$ & 11.33 \\
\hline L inf temporal & 98 & $-45-66-6$ & 11.22 \\
\hline \multicolumn{4}{|l|}{ L sup parietal, } \\
\hline \multicolumn{4}{|l|}{ L inf parietal, } \\
\hline L mid occipital & 376 & $-27-7220$ & 10.03 \\
\hline \multicolumn{4}{|c|}{ CONTROL-CATEGORICALTASK FOR YOUNG AND OLDTOGETHER } \\
\hline Supp motor area & 619 & $-6-963$ & 9.64 \\
\hline R rolandic oper & 299 & 5733 & 9.28 \\
\hline L sup temporal & 264 & $-60-33$ & 7.08 \\
\hline \multicolumn{4}{|c|}{ COORDINATE-CONTROL TASK FOR YOUNG AND OLDTOGETHER } \\
\hline L cerebellum & 345 & $-9-81-30$ & 15.08 \\
\hline \multicolumn{4}{|l|}{$\mathrm{R}$ angular, } \\
\hline \multicolumn{4}{|l|}{ R mid occipital, } \\
\hline R inf temporal & 1963 & $33-7230$ & 14.08 \\
\hline \multicolumn{4}{|l|}{ L inf parietal, } \\
\hline \multicolumn{4}{|l|}{ L sup parietal, } \\
\hline L inf temporal & 1224 & $-30-5448$ & 11.60 \\
\hline L mid frontal & 108 & $-27-651$ & 10.11 \\
\hline \multicolumn{4}{|l|}{ R mid frontal, } \\
\hline R inf frontal & 386 & 453618 & 9.44 \\
\hline R mid frontal & 163 & 30651 & 7.76 \\
\hline
\end{tabular}

CONTROL-COORDINATE TASK FOR YOUNG AND OLDTOGETHER

Mid cingulate,

$\begin{array}{lrrr}\text { post cingulate } & 1752 & -3-3042 & 10.90 \\ \text { L mid frontal } & 911 & -242736 & 9.12 \\ \text { R sup temporal } & 310 & 5703 & 9.10 \\ \text { L mid temporal } & 115 & -63-9-18 & 8.64 \\ \text { L angular } & 143 & -51-7233 & 8.13 \\ \text { R precentral } & 139 & 54-648 & 8.07\end{array}$

COORDINATE-CATEGORICALTASKS FOR YOUNG AND OLDTOGETHER

R mid frontal,

$\begin{array}{lrrr}\text { R insula } & 1041 & 454218 & 10.99 \\ \text { L insula } & 151 & -3318-3 & 10.33 \\ \text { R inf parietal, } & & & \\ \text { R mid occipital, } & & & \\ \text { R angular } & 1154 & 98-4248 & 9.13 \\ \text { Mid cingulate } & 169 & & 8.98 \\ \text { L sup parietal, } & & -18-7248 & \\ \text { L inf parietal } & 349 & -30-66-36 & 8.92 \\ \text { L cerebellum } & 270 & 30651 & 6.21 \\ \text { R mid frontal } & 77 & & \end{array}$

The reverse contrast for task difference (categorical-coordinate) revealed no additional significant clusters. All results reported are thresholded with FWE correction at $p<0.05$.

activated bilateral cerebellum and bilateral prefrontal cortex (left and right middle and inferior frontal gyri, left and right insula, left supplementary motor area).
A direct comparison between the (harder) coordinate task and (easier) categorical task across all the subjects revealed activation in many frontal and parietal sites (Figure 2C and Table 2). These sites included right and left middle frontal gyrus, right and left insula, right and left posterior parietal cortex, and right supplementary motor area. On the other hand, the categorical task, when contrasted with the coordinate task, differentially activated only one significant 9-voxel cluster in posterior cingulate. Furthermore, masking this contrast to show only positive (categorical > control) task-related activation eliminated this cluster. An age (young and old) by task (coordinate and categorical) interaction revealed no significant activation after correcting for multiple comparisons across the whole brain at the $p<0.05$ level. However, when a slightly more liberal criterion was used $(p<0.001$, uncorrected), we found more coordinate vs. categorical activation in left posterior parietal cortex in the old than in the young.

Figure 3 reveals a number of areas where the difference between coordinate and control tasks differed by age group: left medial orbital frontal cortex, left lingual gyrus, and left cuneus (Figure 3C and Table 3). Notably, in most of these areas, the results were due to greater deactivation in the young rather than greater activation in the old (see Figures 3A,B and Table $\mathbf{3}$ to note directionality of age effects).

In summary, analyses of the categorical and coordinate tasks revealed that (a) both the categorical and coordinate tasks led to activity in ventral visual and posterior parietal areas in both the young and old subjects; (b) age effects were generally limited to medial structures deactivated during the coordinate task; (c) the categorical activations were entirely subsumed by the coordinate task, suggesting that the categorical task was not unique from the coordinate task, and (d) the coordinate task required considerably more cognitive activity than the categorical task, with old showing unique activity in the left posterior parietal cortex on this task.

\section{Default network analysis}

In order to study the behavior of the default network in the two age groups, we first identified brain areas that were significantly deactivated by both spatial judgment tasks relative to the control task. Contrasting control vs. (categorical + coordinate) in a group analysis combining young and old subjects revealed a number of areas of activation, mainly in lateral parietal and midline structures. We then applied an anatomical mask (Tzourio-Mazoyer et al., 2002) corresponding to regions associated with canonical default network (Raichle et al., 2001; Greicius et al., 2003) consisting of anterior cingulate and medial prefrontal cortex, precuneus, posterior cingulate and retrosplenial cortex, hippocampus and parahippocampal cortex, and lateral inferior parietal cortex. We identified the three regions that were most activated by the control vs. (categorical + coordinate) contrast within this mask as well as the three regions most activated by the contrast outside the mask. To generate ROIs for these regions, we isolated local maxima and then drew $4 \mathrm{~mm}$ diameter spheres around these points. We then examined mean parameter estimates for both the categorical-control contrast and the coordinate-control contrast within these six regions in both the young and old participants. 


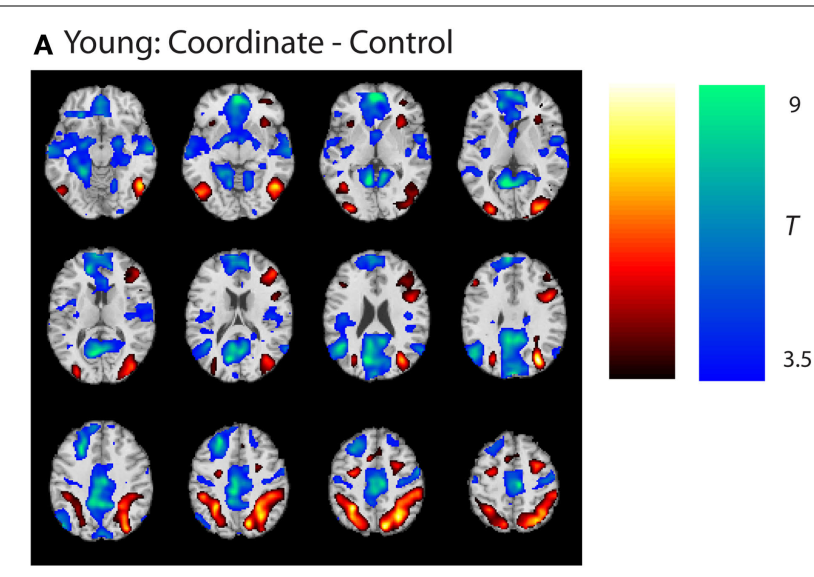

B Old: Coordinate - Control

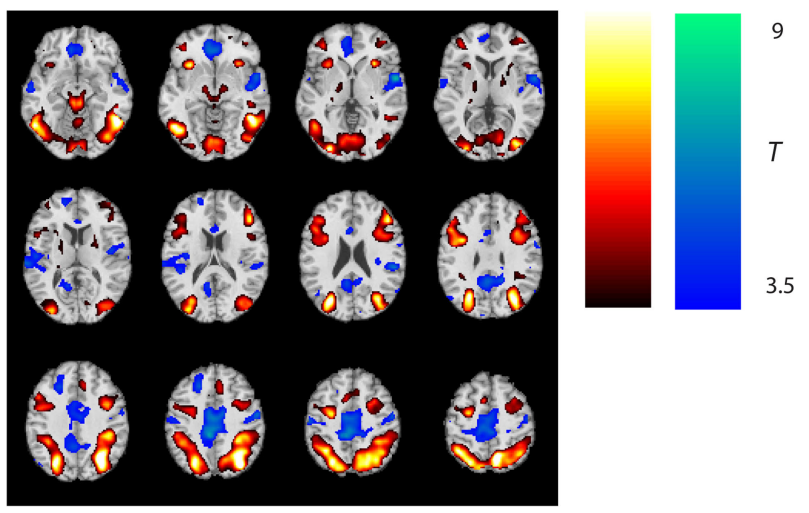

c Young - Old: Coordinate - Control

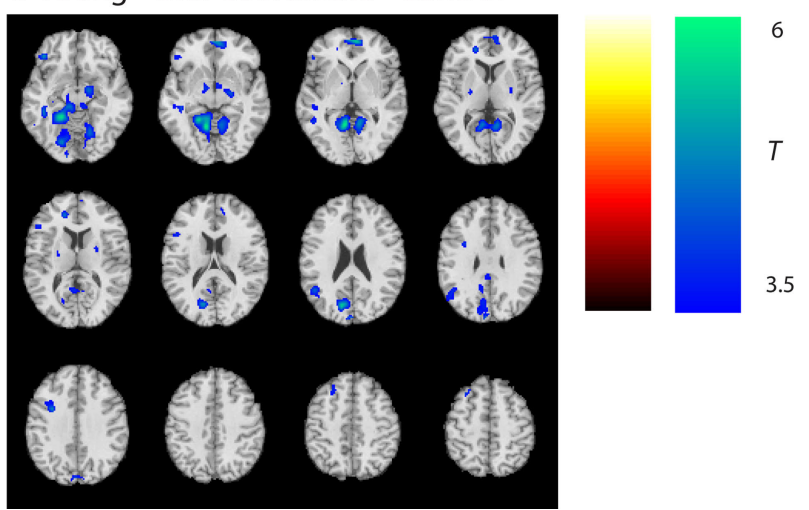

FIGURE 3 | Group statistical maps, age effects. (A) Coordinate-control task T-map for young subjects. (B) Coordinate-control task T-map for old subjects. (C) Coordinate-categorical tasks T-map for young-old (Age $\times$ Task interaction). Note the reverse contrast reveals no significant activation. All maps thresholded at $p<0.001$ with no cluster correction, and reverse contrasts shown in cold colors.

Figure 4 shows the results. In all three regions within the canonical default network (Figure 4, left), we found a significant interaction between Task (categorical vs. coordinate) and Age (young vs. old) [in the anterior cingulate, $F(1,30)=6.48$, $p<0.05$; in the posterior cingulate, $F(1,30)=18.88, p<0.001$; and in the left angular gyrus and $F(1,30)=19.18, p<0.001]$. The young subjects deactivated these areas much more when

Table 3 |Top clusters from group statistical maps, age effects.

\begin{tabular}{lccc}
\hline Region & Cluster extent & MNI mm (peak) & t value (peak) \\
\hline $\begin{array}{l}\text { COORDINATE-CONTROL CONTRAST FOR YOUNG SUBJECTS } \\
\text { R mid occipital, } \\
\text { R inf temporal, }\end{array}$ & & \\
R precuneus & 2044 & $33-7230$ & 9.47 \\
L inf parietal, & & & \\
L sup parietal & 1090 & $-30-5148$ & 8.81 \\
R inf frontal & 533 & 48927 & 6.35 \\
R mid frontal & 197 & 33354 & 6.31 \\
L mid frontal & 108 & $-27-651$ & 6.15 \\
CONTROL-CO &
\end{tabular}

\section{CONTROL-COORDINATE CONTRAST FOR YOUNG SUBJECTS}

L lingual,

supp motor area,

$\begin{array}{llll}\text { L calcarine } & 3233 & -9-573 & 14.83\end{array}$

Med orb frontal,

L mid frontal,

$\begin{array}{llll}\text { L sup frontal } & 1510 & 054-3 & 12.43\end{array}$

$\begin{array}{llll}L & \text { angular } & 315 & -45-5724\end{array}$

R mid temporal,

$\begin{array}{llll}\text { R sup temporal } & 267 & 66-12-12 & 7.72\end{array}$

$\begin{array}{llll}\text { R precentral } & 138 & 54-351 & 7.58\end{array}$

R amygdala,

R hippocampus,

$\begin{array}{llll}R \text { parahippo } & 160 & 330-21 & 7.21\end{array}$

COORDINATE-CONTROL CONTRAST FOR OLD SUBJECTS

$\mathrm{R}$ mid occipital,

R sup occipital,

$R$ precuneus,

$\begin{array}{lrrr}\text { R inf temporal } & 6710 & -9-81-30 & 15.78 \\ \text { R insula } & 112 & 3021-3 & 9.65 \\ \text { L mid frontal, } & & & \\ \text { L inf frontal } & 787 & -27-651 & 8.96 \\ \text { R inf frontal, } & & & \\ \text { R mid frontal } & 928 & 483321 & 8.87 \\ \text { Supp motor area } & 133 & -61251 & 6.43\end{array}$

CONTROL-COORDINATE CONTRAST FOR OLD SUBJECTS

Mid cingulate,

$\begin{array}{lrrr}\text { post cingulate } & 1684 & -6-3345 & 7.65 \\ \text { R temporal pole } & 314 & 5730 & 7.60 \\ \text { R precentral gyrus } & 220 & 54-945 & 6.03 \\ \text { Anterior cingulate } & 297 & 339-6 & 5.84 \\ \text { L supramarginal } & & & \\ \text { gyrus } & 209 & -66-2415 & 5.83 \\ \text { L sup frontal } & 122 & -183042 & 5.67\end{array}$

\section{AGE $\times$ TASK INTERACTION FOR COORDINATE-CONTROL} CONTRAST

L lingual,

L cuneus,

$\mathrm{L}$ cerebellum

1858

Med orb frontal,

Med sup frontal

L orb inf frontal

L angular,

L mid temporal

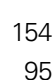

$$
-9-57-6
$$

$$
057-3
$$$$
-4236-12
$$

4.96

Interaction effects are primarily driven by greater control-related activation in young. All results thresholded at $p<0.001$ with no cluster correction. 
Table 4 | Pearson's $r$ correlations between time series residuals in default regions in both young $(\mathrm{Y})$ and old $(\mathrm{O})$.

\begin{tabular}{|c|c|c|c|c|c|c|}
\hline & mPFC & pC & HF & PHC & IIP & rIP \\
\hline \multirow[t]{2}{*}{ mPFC } & & $Y=0.19$ & $Y=0.01$ & $Y=0.01$ & $Y=0.06$ & $Y=0.05$ \\
\hline & & $\mathrm{O}=0.29$ & $O=0.02$ & $O=0.09$ & $O=0.13$ & $\mathrm{O}=0.23 * *$ \\
\hline \multirow[t]{2}{*}{$\mathrm{pC}$} & & & $Y=0.37$ & $Y=0.32$ & $Y=0.39$ & $Y=0.25$ \\
\hline & & & $O=0.20$ & $O=0.18$ & $\mathrm{O}=0.31$ & $O=0.33$ \\
\hline \multirow[t]{2}{*}{$\mathrm{HF}$} & & & & $Y=0.43$ & $Y=0.29 * *$ & $Y=0.21 * *$ \\
\hline & & & & $O=0.27$ & $\mathrm{O}=0.06$ & $\mathrm{O}=0.04$ \\
\hline \multirow[t]{2}{*}{$\mathrm{PHC}$} & & & & & $Y=0.32 * *$ & $Y=0.22$ \\
\hline & & & & & $\mathrm{O}=0.10$ & $\mathrm{O}=0.14$ \\
\hline \multirow[t]{2}{*}{$\| P$} & & & & & & $Y=0.46$ \\
\hline & & & & & & $\mathrm{O}=0.35$ \\
\hline $\mathrm{rIP}$ & & & & & & \\
\hline
\end{tabular}

Areas include medial prefrontal (mPFC), posterior cingular cortex ( $p C)$, hippocampal formation (HF), parahippocampal cortex (PHC), and left and right lateral parietal lobe (IIP and rIP, respectively).

**Indicates a statistically significant difference between the two age groups after correcting for 15 multiple comparisons.

performing the harder coordinate task than when performing the easier categorical task, but the old subjects did not exhibit this pattern.

Interestingly, this Age $\times$ Task interaction was specific to the default network and was not observed in areas deactivated by the tasks that fell outside the canonical network (Figure 4, right). In the three areas outside this network that were most deactivated by the tasks, the interactions did not approach significance. These areas were the supplementary motor area $[F(1,30)=0.43, p=0.52]$, the middle cingulate, $[F(1,30)=0.68$, $p=0.42]$, and the right rolandic operculum $[F(1,30)=0.018$, $p=0.90]$. Furthermore, when the data from these three areas were combined and compared with the combined data from the three areas within the default network, we confirmed that the Age $\times$ Task interaction was significantly larger in the default network than outside it (that is, the three-way interaction among Age, Task, and Brain Area (in or out of default network) was highly significant $[F(1,30)=18.44, p<0.001]$. Since age group was confounded with level of performance on the imaging tasks (i.e., older subjects were slower on both tasks and less accurate on the coordinate task), these tested models included RT and accuracy as nuisance regressors.

One particularly interesting aspect of these results is that the Age $\times$ Task interaction was observed in the anterior and posterior cingulate, but not in the middle cingulate. We therefore analyzed the significance of the interaction throughout the cingulate. Figure 5 shows the results of computing a $t$-value corresponding to a difference of differences: the difference between the age groups in the size of the difference between the two tasks' deactivation. As the figure illustrates, there appears to be a continuum along the cingulate in the size of this Age $\times$ Task interaction. The young deactivate the anterior and posterior ends much more for the harder coordinate task than for the easier categorical task, but this is not true for the old. This difference between the age groups disappears and, if anything, slightly reverses in the middle cingulate.

\section{Connectivity analysis}

Andrews-Hanna et al. (2007) found that elderly subjects exhibited a significant disruption in connectivity between areas in the default network relative to younger subjects. We found mixed support for this prediction in our dataset, using a connectivity analysis based on correlating filtered time series. Table 4 presents all 15 pairwise correlations between the six default areas from Andrews-Hanna et al. (2007) in both age groups. Most of the correlations are larger in the young subjects than in the old subjects. In particular, 9 out of 10 correlations that did not involve the medial prefrontal cortex were larger in the young. Three of these differences reached statistical significance after correcting for 15 multiple comparisons, all involving connectivity between medial temporal and parietal cortex. On the other hand, connectivity between medial prefrontal cortex and all five other default regions tended to be stronger in the old subjects than in the young with one of these five differences reaching significance.

\section{Correlations with behavior}

Wig et al. (2008) reported that individuals who exhibited greater task-induced deactivations in the medial temporal lobe during encoding tended to show better subsequent memory performance. To examine in our own data whether default suppression enhanced cognitive performance, we computed the average task-induced deactivation (the difference in betas between the control condition and the combined coordinate and categorical conditions) in the same six default regions that we considered in the connectivity analysis in each subject. We then correlated these average deactivation measures with reaction time on the coordinate and categorical tasks (Figure 6). We found that the effect of task-induced deactivation on performance depended on age: Specifically, there was a negative, though non-significant, correlation between RT and average taskinduced deactivation in the default network in the old (categorical task: $r=-0.14, p=0.56$; coordinate task: $r=-0.31, p=0.19$ ). That is, older subjects who deactivated the default network more during task performance performed somewhat better (faster RT, though non-significant). In contrast, we found significant positive correlations between these measures in the young (categorical task: $r=0.53, p<0.05$; coordinate task: $r=0.47, p<0.05)$. This interaction between age and amount of default deactivation was significant for the coordinate task $[F(1,34)=5.47, p<0.03]$ but not for the categorical task $[F(1,34)=2.87, p<0.10]$. Finally, to be more comparable to Wig et al. (2008), we also performed the same analysis restricted to medial temporal sites. Again, older subjects who exhibited more task-induced deactivation performed better (faster RT) (categorical task: $r=-0.46, p<0.05$; coordinate task: $r=-0.55$, $p<0.02$ ) while younger subjects who exhibited more task-induced deactivation performed worse (categorical task: $r=0.55, p<0.02$; coordinate task: $r=0.39, p<0.10$ ) and the age $\times$ default deactivation was highly significant [categorical task: $F(1,34)=10.99, p<0.002$; coordinate task: $F(1,34)=10.38, p<0.003]$. Note that although we chose an ROI approach to observe these age difference in the $\mathrm{RT} \times$ deactivation relationship, a whole-brain analysis of the interaction among age, RT, and deactivation produced a significant result in medial frontal, parietal, and MTL regions commonly associated with the default network. In each of these areas, the relationship between increased deactivation and faster performance is positive for old and negative for young, replicating the ROI findings. 

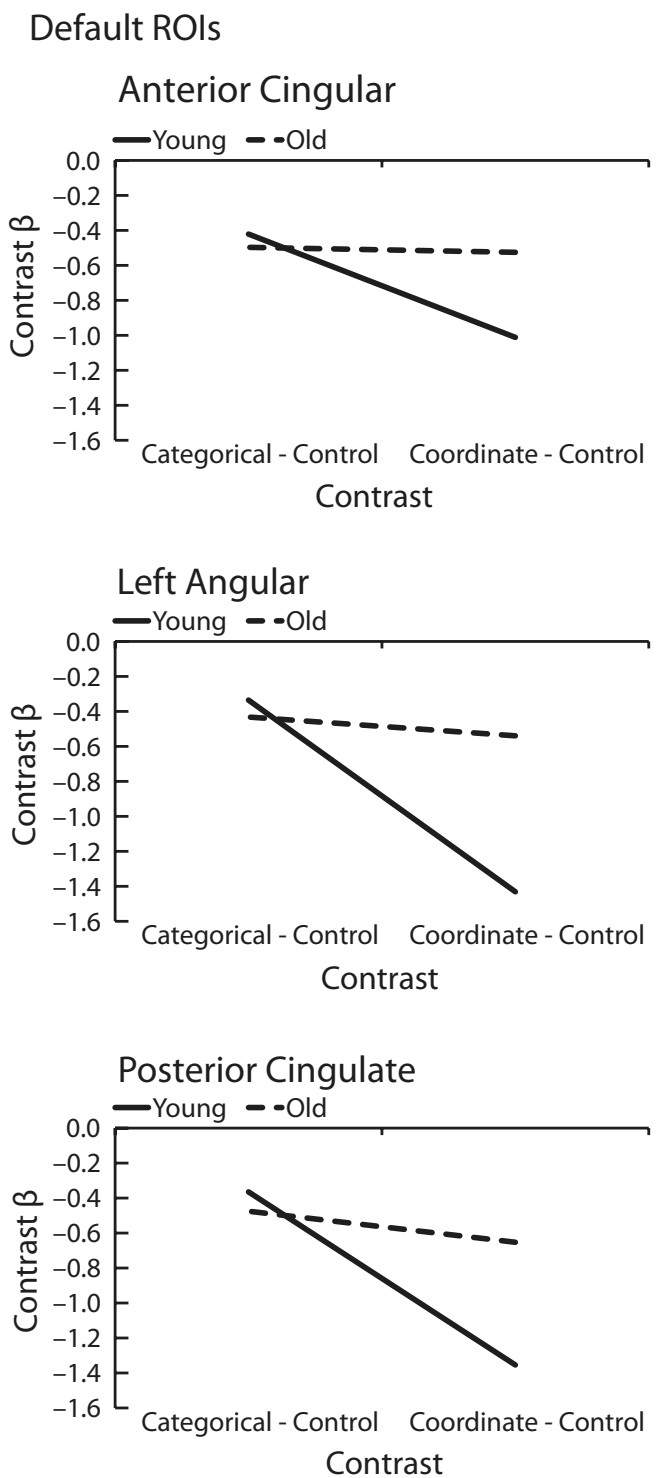
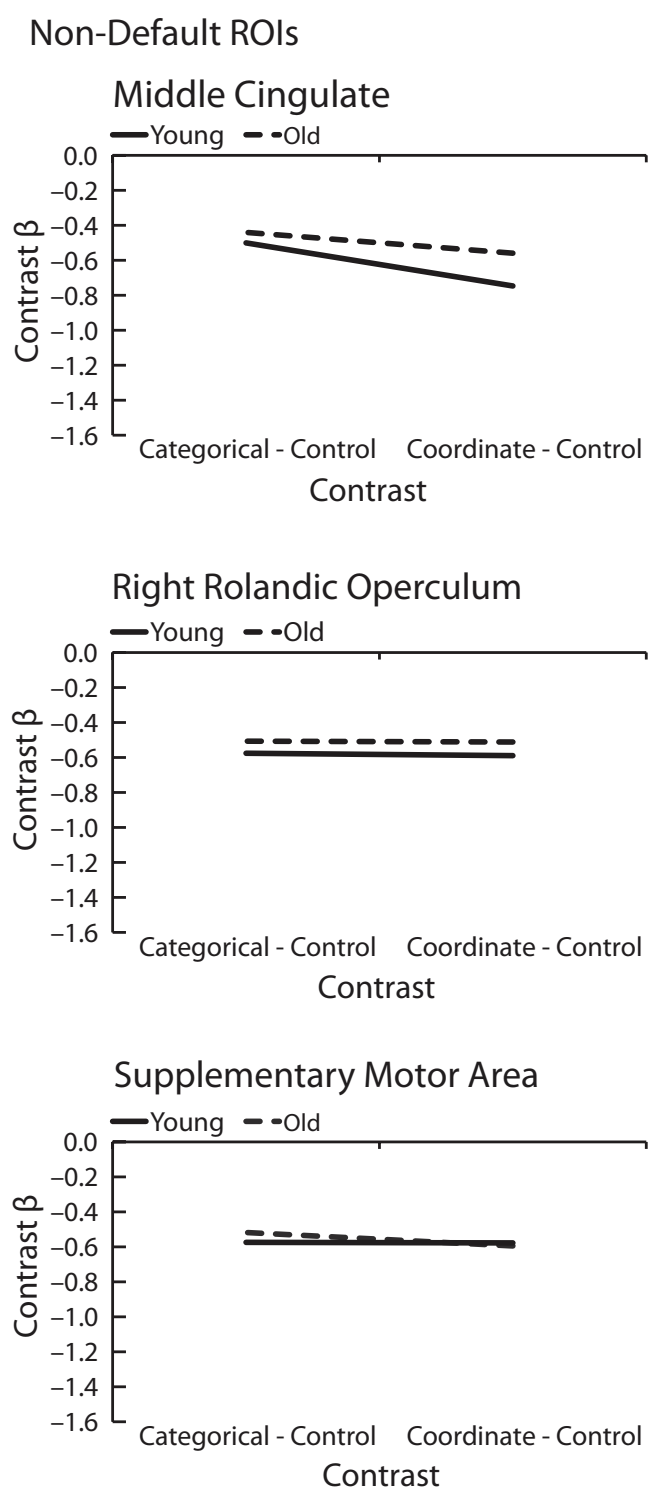

FIGURE 4 | Age differences in categorical and coordinate deactivations in default and non-default ROls. Contrast betas for young and old groups were extracted from ROls defined by a control-task contrast.

\section{DISCUSSION}

The present study investigated age differences in task-related deactivation in two spatial tasks that varied in difficulty, specifically within areas commonly associated with default mode activity. We found that old and young showed a low and comparable level of default suppression on the easy categorical task, but young adults showed increasing suppression of default activity on the more demanding coordinate task. Older adults, however, showed no such modulation of default activity as the task became more difficult. This finding is similar to the only other study in the literature that examines age differences in default activity as a function of task demands. Persson et al. (2007) studied default activity on a semantic verbal retrieval task that varied in effort, and reported default modulation with effort by young but not old. The present findings suggest that this difficulty in default modulation extends to the spatial domain and suggests that it may be a global age-related phenomenon.

Importantly, our findings indicate that this differential effect of task difficulty depending on age is specific to default sites and is not present in deactivated brain regions that are outside the classically observed default network. Indeed, the differing pattern for default vs. non-default sites was elegantly demonstrated within the three regions of the cingulate gyrus (see Figure 5). The anterior and posterior portions of the cingulate are part of the default network and showed increased suppression under high task demands in the young but not the old. However, the middle cingulate is outside the default network, and there was 

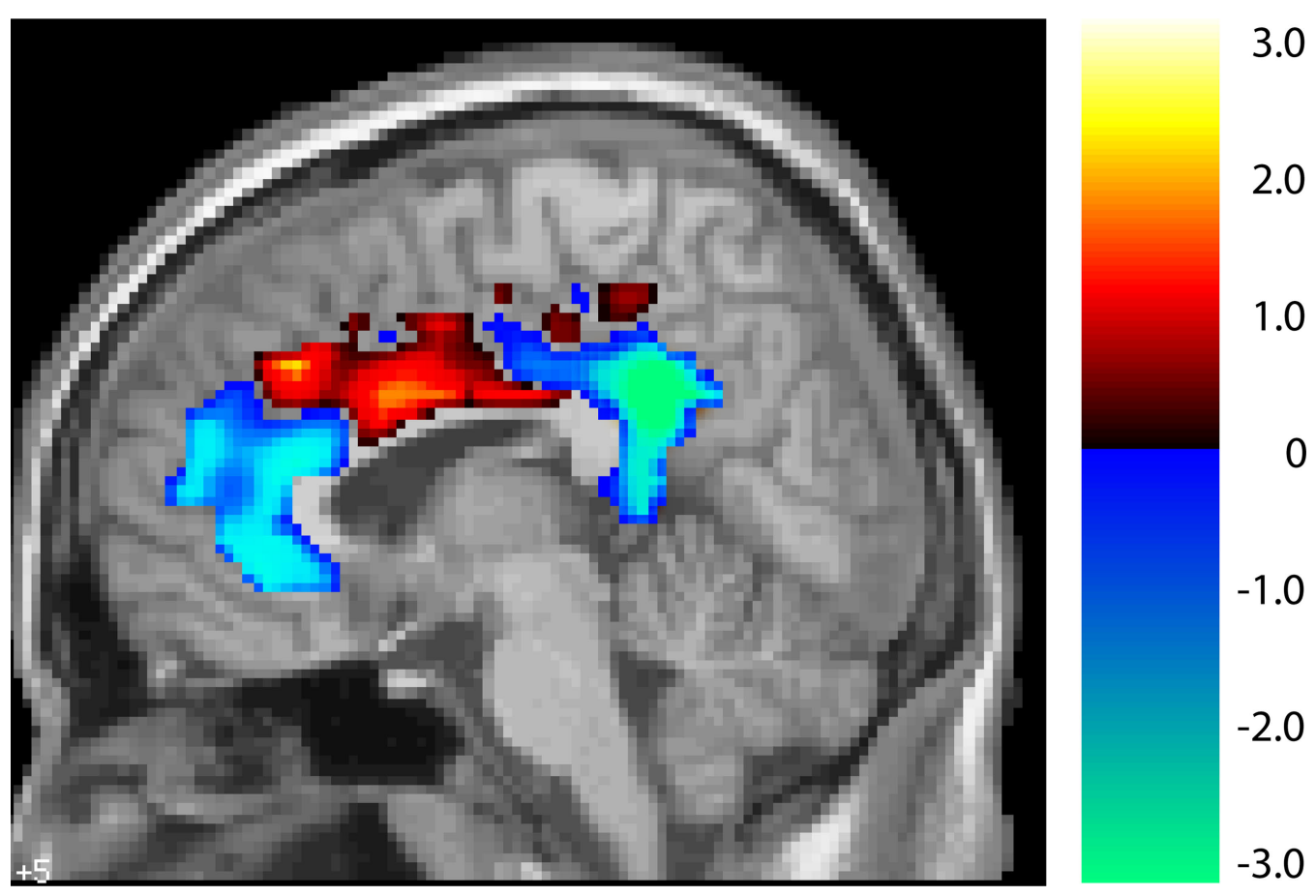

FIGURE 5 | T-map of age by task interaction along the cingulate (unthresholded). In blue areas, young show greater suppression for the harder coordinate task while old show no such task-related differential deactivation. In red areas there are no significant differences between young and old.

no Age $\times$ Task interaction observed in this area, indicating both the specificity of the effect in young adults and the general failure at modulation in old.

We also report evidence for differences in connectivity in older adults compared to young. Our findings provide some evidence for greater connectivity in young compared to old, but primarily between mediotemporal and parietal regions. Of particular note, we report evidence for somewhat greater connectivity in old compared to young for medial prefrontal cortex to other regions, particularly to the right lateral parietal cortex. One explanation for the present pattern is that young rely on greater posterior connectivity, consistent with work that suggests younger adults have more efficient processing in posterior areas (Davis et al., 2008). The young reliance on posterior sites is also broadly consistent with findings from our own lab showing poor neural selectivity in old in ventral visual sites, including the parahippocampus (Park et al., 2004) and decreased activation in hippocampal sites (Gutchess et al., 2005). In contrast to young, old rely on functional connections that are more anterior when performing this visuo-spatial task, consistent with the notion of compensatory activations residing in frontal areas for deficient mediotemporal and ventral visual activations (Park and Reuter-Lorenz, 2009).

Andrews-Hanna et al. (2007) reported that older adults showed a significantly weaker anterior-posterior correlation than young, with old showing no relationship $(r=0.00)$. Even nine amyloidnegative subjects showed no relationship $(r=0.03)$ between these regions. In contrast, the older subjects in our study exhibited somewhat stronger connectivity between anterior (mPFC) and posterior sites compared with the young. Perhaps the differences between our findings and Andrews-Hanna et al. (2007) reflect a modulation of default network connectivity as a function of task type. Andrews-Hanna et al. (2007) used a verbal semantic classification task that has been widely used in the imaging literature (e.g., Logan et al., 2002; Lustig and Buckner, 2004) whereas we used a visuo-spatial judgment task that seems to depend on parietal cortex. The disparity between the results of these studies suggests that age-related changes in default connectivity may depend on the task and points to the need for studies of connectivity within subjects across tasks to investigate the extent to which default connectivity is invariant within a subject or dependent on variations in conditions.

We also found that there are consequences of default activity for behavior. When we examined the performance of older adults, we found a trend such that those who showed the most default suppression on the difficult coordinate task showed the fastest reaction times (see Figure 6). Wig et al. (2008) reported a similar finding in young adults who were performing a memory task, reporting that subjects who exhibited the most suppression of the default network also exhibited the best performance in an offline memory task. The surprising finding in our data was that for young adults, the faster participants showed less suppression of default sites. This was not what we expected but the relationship is strong and reliable across ROIs. Reaction times on the task for young adults were well under $1000 \mathrm{~ms}$ on the task and the presentation interval was $2000 \mathrm{~ms}$. We suspect that fast young subjects returned to the default state as soon as they completed their response, thereby 


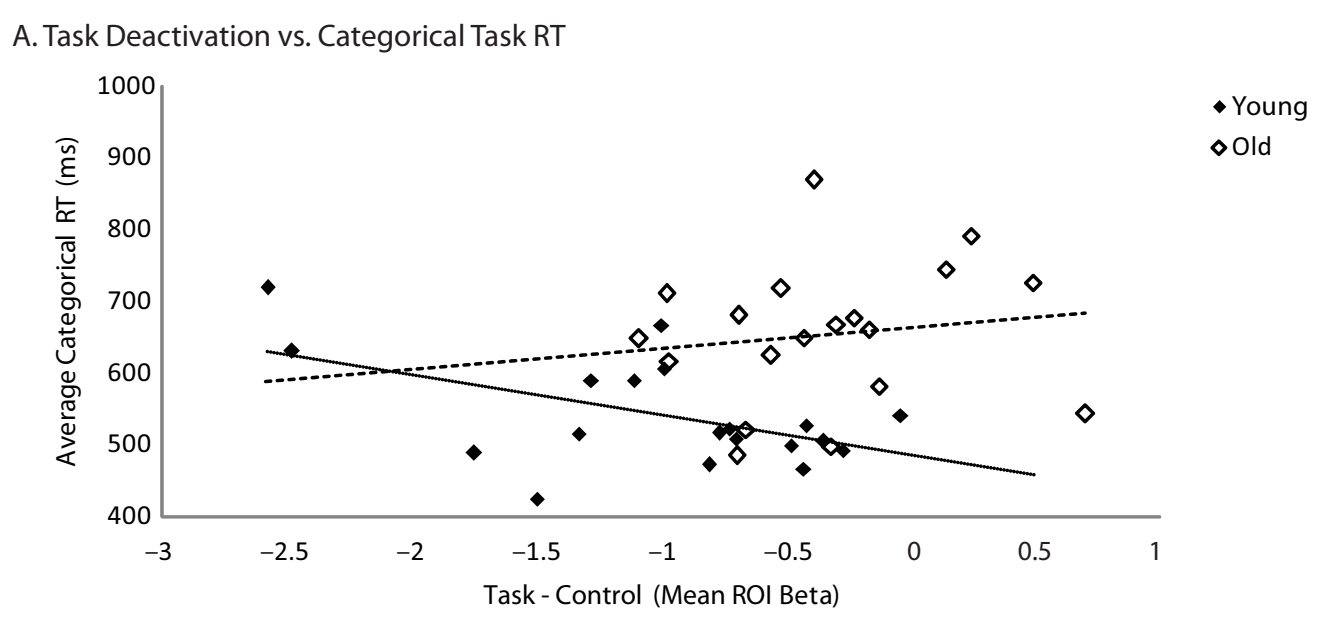

B. Task Deactivation vs. Coordinate Task RT

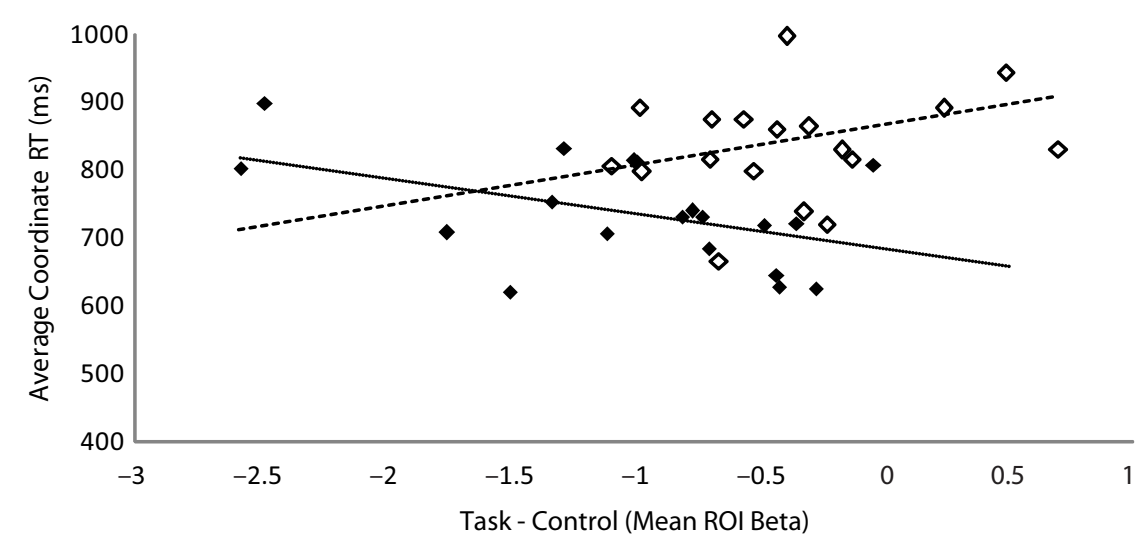

- Young

$\diamond$ Old

FIGURE 6 | Age differences in the correlation between task-related deactivation and task RT. (A) Categorical task RT as a function of task-control contrast betas averaged across default ROIs (medial prefrontal, posterior cingulate, hippocampus, parahippocampal cortex, and left and right parietal cortex). (B) Coordinate task RT as a function of task-control contrast betas averaged across same ROls. showing, on average, more default mode activity during a task block. We recognize this to be a speculative interpretation worthy of further study. What is clear from the present data is that default activity is important for performance, and that much more work is needed to delineate the exact nature of these relationships. Nevertheless, the finding that older adults were slower when they failed to suppress the default network suggests that for them at least poor cognitive performance is related to inefficient default modulation, in agreement with Andrews-Hanna et al. (2007) and Damoiseaux et al. (2008). There are multiple potential causes of the relationship between poor cognitive function and poor default modulation. At present, estimates are that $30 \%$ of normal elderly have amyloid deposition (Rodrigue et al., 2009) Thus it is virtually certain that some of the older adults in the present study are carrying a significant amyloid burden, and it is a possibility that amyloid deposition in slower adults in this study may be interfering with the performance of the default network. In a recent model of neurocognitive aging (Scaffolding Theory of Aging and Cognition), Park and Reuter-Lorenz (2009) explicitly note that both inefficient default activation and high amyloid burden are likely to be characteristic of poor cognitive function. There is also growing evidence that decreased vascular health is associated with decreased neural health (Colcombe et al., 2004) and this mechanism is also a potential basis for the observed relationship between speed in older adults and default activity. Overall, there is still much that remains to be learned about the relationship of default activity to exogenous factors and behavioral function.

The other findings from the study relate to observed activations, rather than deactivations. We found that the easy categorical judgment task engaged bilateral posterior parietal cortex in both young and old adults and yielded no significant differences with age. When performing the more demanding coordinate task, however, prefrontal and additional parietal resources were engaged by both groups. We note that, categorical task activation was entirely subsumed by coordinate task activation. The data provide strong evidence that the categorical task was largely performed with a subset of sites used for the coordinate task, and that the coordinate task was much more demanding. The reaction time and error data also support this conclusion. There is a literature suggesting that coordinate tasks strongly activate left parietal cortex, a finding 
consistent with our own data, as shown in Figure 2B. There is also mixed evidence regarding the possibility that the categorical task sometimes loads more on right parietal cortex (Kosslyn et al., 1998; Baciu et al., 1999). The data on this are inconsistent and, like other studies (Jager and Postma, 2003; Martin et al., 2008), we found little evidence for right parietal specificity.

In sum, our major findings suggest that age-related changes in the modulation of default mode processing play an important role in age-related cognitive impairments. Older adults have difficulty suppressing the default network and this impairment is particularly apparent on difficult tasks. Furthermore, these effects appear to be task-independent, being observed in both verbal memory tasks and in non-verbal spatial tasks. Nevertheless, they are specific to the default network and are not observed in other sites that were deactivated by the tasks. We also found that functional connectivity

\section{REFERENCES}

Addis, D. R., Wong, A. T., and Schacter, D. L. (2008). Age-related changes in the episodic simulation of future events. Psychol. Sci. 19, 33-41.

Andrews-Hanna, J. R., Snyder, A. Z., Vincent, J. L., Lustig, C., Head, D., Raichle, M. E., and Buckner, R. L. (2007). Disruption of large-scale brain systems in advanced aging. Neuron 56 , 924-935.

Baciu, M., Koenig, O., Vernier, M. P., Bedoin, N., Rubin, C., and Segebarth, C. (1999). Categorical and coordinate spatial relations: fMRI evidence for hemispheric specialization. Neuroreport 10, 1373-1378.

Boly, M., Tshibanda, L., Vanhaudenhuyse, A., Noirhomme, Q., Schnakers, C., Ledoux, D., Boveroux, P., Garweg, C., Lambermont, B., Phillips, C., Luxen, A., Moonen, G., Bassetti, C., Maquet, P., and Laureys, S. (2009). Functional connectivity in the default network during resting state is preserved in a vegetative but not in a brain dead patient. Hum. Brain Mapp. 30, 2393-2400.

Buckner, R. L., Snyder, A. Z., Shannon, B. J., LaRossa, G., Sachs, R., Fotenos, A. F., Sheline, Y. I., Klunk, W. E., Mathis, C. A., Morris, J. C., and Mintun, M. A. (2005). Molecular, structural, and functional characterization of Alzheimer's disease: evidence for a relationship between default activity, amyloid, and memory. J. Neurosci. 25, 7709-7717.

Cabeza, R. (2002). Hemispheric asymmetry reduction in older adults: the HAROLD model. Psychol. Aging 17, 85-100.

Cabeza, R., Anderson, N. D., Locantore, J. K., and McIntosh, A. R. (2002). Aging gracefully: compensatory brain activity in high-performing older adults. Neuroimage 17, 1394-1402.

Chee, M. W., Goh, J. O., Venkatraman, V., Tan, J. C., Gutchess, A., Sutton, B.,
Hebrank, A., Leshikar, E., and Park, D. C. (2006). Age-related changes in object processing and contextual binding revealed using fMR adaptation. J. Cogn. Neurosci. 18, 495-507.

Colcombe, S. J., Kramer, A. F., Erickson, K. I., Scalf, P., McAuley, E., Cohen, N. J., Webb, A., Jerome, G. J., Marquez, D. X., and Elavsky, S. (2004). Cardiovascular fitness, cortical plasticity, and aging. Proc. Natl. Acad. Sci. U.S.A. 101, 3316-3321.

Damoiseaux, J.S., Beckmann, C. F., Arigita, E. J. S., Barkhof, F., Scheltens, P., Stam, C. J., Smith, S. M., and Rombouts, S. A. R. B. (2008). Reduced resting-state brain activity in the "default network" in normal aging. Cereb. Cortex 18, 1856-1864.

Davis, S. W., Dennis, N. A., Daselaar, S. M., Fleck, M.S., and Cabeza, R. (2008). Que PASA? The posterior-anterior shift in aging. Cereb. Cortex 18 , 1201-1209.

Duverne, S., Motamedinia, S., and Rugg, M. D. (2009). The relationship between aging, performance, and the neural correlates of successful memory encoding. Cereb. Cortex 19, 733-744.

Folstein,M.F., Folstein, S.E., and McHugh, P. R. (1975). "Mini-mental state". A practical method for grading the cognitive state of patients for the clinician. J Psychiatr. Res 12, 189-198. Corbetta, M., Van Essen, D. C., and Raichle, M. E. (2005). The human brain is intrinsically organized into dynamic, anticorrelated functional networks. Proc. Natl. Acad. Sci. U.S.A. 102, 9673-9678.

Grady, C. L., Springer, M. V., Hongwanishkul, D., McIntosh, A. R., and Winocur, G. (2006). Age-related changes in brain activity across the adult lifespan. J. Cogn. Neurosci. 18, 227-241.

Greicius, M. D., Kiviniemi, V., Tervonen, O., Vainionpää, V., Alahuhta, S., Reiss,
Fox, M. D., Snyder, A. Z., Vincent, J. L.,

among posterior regions of the default network declines with age but that functional connectivity with medial prefrontal cortex increases, consistent with recent findings of a posterior-anterior shift in aging (Davis et al., 2008) but in contrast to findings of age-related declines in anterior-posterior connectivity in a verbal memory paradigm (Andrews-Hanna et al., 2007). Finally, the ability to modulate activity in the default network has behavioral implications: Older adults who succeed in suppressing the default network tend to perform better.

\section{ACKNOWLEDGMENTS}

We gratefully acknowledge the members of the Center for Vital Longevity for valuable discussions regarding this project and paper. This work was supported by grant 5R37AG006265-25 from the National Institute on Aging.

A. L., and Menon, V. (2008). Persistent default-mode network connectivity during light sedation. Hum. Brain Mapp. 29, 839-847.

Greicius, M. D., Krasnow, B., Reiss, A L., and Menon, V. (2003). Functional connectivity in the resting brain: a network analysis of the default mode hypothesis. Proc. Natl. Acad. Sci. U.S.A. 100, 253-258.

Greicius, M. D., Srivastava, G., Reiss, A. L., and Menon, V. (2004). Defaultmode network activity distinguishes Alzheimer's disease from healthy aging: evidence from functional MRI. Proc. Natl. Acad. Sci. U.S.A. 101, 4637-4642.

Gutchess, A. H., Welsh, R. C., Hedden, T., Bangert, A., Minear, M., Liu, L. L., and Park, D. C. (2005). Aging and the neural correlates of successful picture encoding: frontal activations compensate for decreased medialtemporal activity. J. Cogn. Neurosci. 17, 84-96.

Jager, G., and Postma, A. (2003). On the hemispheric specialization for categorical and coordinate spatial relations: a review of the current evidence. Neuropsychologia 41, 504-515.

Kosslyn, S. M., Thompson, W. L., Gitelman, D. R., and Alpert, N. M. (1998). Neural systems that encode categorical versus coordinate spatial relations: PET investigations. Psychobiology 26, 333-347.

Logan, J. M., Sanders, A. L., Snyder, A. Z., Morris, J. C., and Buckner, R. L. (2002). Under-recruitment and nonselective recruitment: dissociable neural mechanisms associated with aging. Neuron 33, 827-40.

Lustig, C., and Buckner, R. L. (2004). Preserved neural correlates of priming in old age and dementia. Neuron $42,865-875$.

Lustig, C., Snyder, A. Z., Bhakta, M., O’Brien, K. C., McAvoy, M., Raichle, M. E., Morris, J. C., and Buckner, R.
L. (2003). Functional deactivations: change with age and dementia of the Alzheimer type. Proc. Natl. Acad. Sci. U.S.A. 100, 14504-14509.

Madden, D. J., Turkington, T. G., Provenzale, J. M., Denny, L. L., Hawk, T. C., Gottlob, L. R., and Coleman, R. E. (1999). Adult age differences in the functional neuroanatomy of verbal recognition memory. Hum. Brain Mapp. 7, 115-135.

Martin, R., Houssemand, C., Schiltz, C., Burnod, Y., and Alexandre, F. (2008). Is there continuity between categorical and coordinate spatial relations coding: evidence from a grid/nogrid working memory paradigm. Neuropsychologia 46, 576-594.

Mason, M. F., Norton, M. I., Van Horn, J. D., Wegner, D. M., Grafton, S. T., and Macrae, C. N. (2007). Wandering minds: the default network and stimulus-independent thought. Science 315, 393-395.

McKiernan, K. A., Kaufman, J. N., KuceraThompson, J., and Binder, J. R. (2003). A parametric manipulation of factors affecting task-induced deactivation in functional neuroimaging. J. $\operatorname{Cog} n$. Neurosci. 15, 394-408.

Miller, S. L., Celone, K., DePeau, K., Diamond, E., Dickerson, B. C., Rentz, D., Pihlajamäki, M., and Sperling, R.A. (2008). Age-related memory impairment associated with loss of parietal deactivation but preserved hippocampal activation. Proc. Natl. Acad. Sci. U.S.A. 105, 2181-2186.

Park, D. C., Lautenschlager, G., Hedden, T., Davidson, N. S., Smith, A. D., and Smith, P. K. (2002). Models of visuospatial and verbal memory across the adult life span. Psychol. Aging 17, 299-320.

Park, D. C., Polk, T. A., Park, R., Minear, M., Savage, A., and Smith, M. R. (2004). Aging reduces neural specialization in ventral visual cortex. Proc. Natl. Acad. Sci. U.S.A. 101, 13091-13095. 
Park, D. C., and Reuter-Lorenz, P. A. (2009). The adaptive brain: aging and neurocognitive scaffolding. Annu. Rev. Psychol. 60, 173-196.

Persson, J., Lustig, C., Nelson, J. K., and Reuter-Lorenz, P. A. (2007). Age differences in deactivation: a link to cognitive control? J. Cogn. Neurosci. 19, 1021-1032.

Raichle, M.E., MacLeod, A. M., Snyder,A. Z., Powers, W. J., Gusnard, D. A., and Shulman, G. L. (2001). A default mode of brain function. Proc. Natl. Acad. Sci. U.S.A. 98, 676-682.

Raichle, M. E., and Snyder, A. Z. (2007), A default mode of brain function: a brief history of an evolving idea. Neuroimage 37, 1083-1090.

Reuter-Lorenz, P. A., Marshuetz, C., Jonides, J., Smith, E. E., Hartley, A., and Koeppe, R. (2001). Neurocognitive ageing of storage and executive processes. Eur. J. Cogn. Psychol. 13, 257.

Rodrigue, K. M., Kennedy, K. M., and Park, D. C. (2009). Beta-amyloid deposition and the aging brain. Neuropsychol. Rev. $19,436-450$.
Rosen, V. M., Bergeson, J. L., Putnam, K., Harwell, A., and Sunderland, T. (2002). Working memory and apolipoprotein E: what's the connection? Neuropsychologia 40, 2226-2233.

Rossi, S., Miniussi, C., Pasqualetti, P., Babiloni, C., Rossini, P. M., and Cappa, S. F. (2004). Age-related functional changes of prefrontal cortex in long-term memory: a repetitive transcranial magnetic stimulation study. J. Neurosci. 24, 7939-7944.

Schacter, D. L., Addis, D. R., and Buckner, R. L. (2007). Remembering the past to imagine the future: the prospective brain. Nat. Rev. Neurosci. 8, 657-661.

Shulman, G. L., Fiez, J. A., Corbetta, M., Buckner, R. L., Miezin, F. M., Raichle, M. E., and Petersen, S. E. (1997). Common blood flow changes across visual tasks: II. Decreases in cerebral cortex. J. Cogn. Neurosci. 9, 648-663.

Tzourio-Mazoyer, N., Landeau, B., Papathanassiou, D., Crivello, F., Etard, O., Delcroix, N., Mazoyer, B., and Joliot, M. (2002). Automated anatomical labeling of activations in SPM using a macroscopic anatomical parcellation of the MNI MRI single-subject brain. Neuroimage 15, 273-289.

Vincent, J. L., Patel, G. H., Fox, M. D., Snyder, A. Z., Baker, J. T., Van Essen, D. C., Zempel, J. M., Snyder, L. H. Corbetta, M., and Raichle, M. E. (2007). Intrinsic functional architecture in the anaesthetized monkey brain. Nature 447, 83-86.

Vincent, J. L., Snyder, A. Z., Fox, M. D., Shannon, B. J., Andrews, J. R., Raichle, M. E., and Buckner, R. L. (2006). Coherent spontaneous activity identifies a hippocampal-parietal memory network. J. Neurophysiol. 96, 3517-3531.

Wechsler, D. (1981). Wechsler Adult Intelligence Scale-Revised.Cleveland, $\mathrm{OH}$, Psychological Corporation.

Wig, G. S., Grafton, S. T., Demos, K. E., Wolford, G. L., Petersen, S. E., and Kelley, W. M. (2008). Medial temporal lobe BOLD activity at rest predicts individual differences in memory ability in healthy young adults. Proc. Natl. Acad. Sci. U.S.A. 105, 18555-18560.
Zachary, R. A. (1986). Shipley Institute of Living Scale, Revised Manual. Los Angeles, Western Psychological Services.

Conflict of Interest Statement: The authors declare that the research was conducted in the absence of any commercial or financial relationships that could be construed as a potential conflict of interest.

Received: 02 September 2009; paper pending published: 05 October 2009; accepted: 22 December 2009; published online: 19 January 2010.

Citation:ParkDC, PolkTA, HebrankACand Jenkins LJ (2010) Age differences in default mode activity on easy and difficult spatial judgment tasks. Front. Hum. Neurosci. 3:75. doi:10.3389/neuro.09.075.2009

Copyright $\odot 2010$ Park, Polk, Hebrank and Jenkins. This is an open-access article subject to an exclusive license agreement between the authors and the Frontiers Research Foundation, which permits unrestricted use, distribution, and reproduction in any medium, provided the original authors and source are credited. 